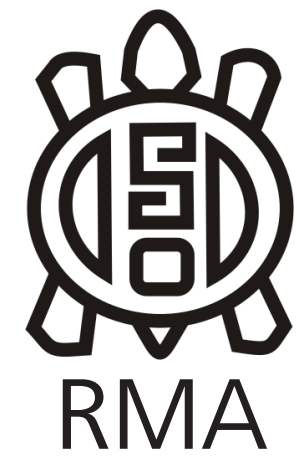

Dossier

\title{
Una aproximación a la distribución de la producción laminar en Patagonia meridional
}

\author{
An approach to the distribution of blade production in southern \\ Patagonia
}

\author{
María Cecilia Pallo*, Natalia Andrea Cirigliano**, Judith Charlin ${ }^{* * *}$ y \\ Karen Borrazzo***
}

*CONICET, Instituto Patagónico de Ciencias Sociales y Humanas, FFyL - UBA, Argentina. E-mail: ceciliapallo@gmail.com ${ }^{* *}$ CONICET, Instituto Multidisciplinario de Historia y Ciencias Humanas, Argentina. E-mail: naticirigliano@hotmail.com *** CONICET, Instituto Multidisciplinario de Historia y Ciencias Humanas, FFyL - UBA, Argentina. E-mail: judith.charlin@gmail.com ; kborrazzo@yahoo.com.ar

\section{Resumen}

Se presenta una primera aproximación a la distribución de la producción laminar manufacturada en lutita al sur de la sierra Baguales (Patagonia sur continental, entre Argentina y Chile). Si bien las láminas son características del área noroccidental al núcleo poblacional identificado en el campo volcánico Pali Aike (Última Esperanza en Chile, y la zona circundante a sierra Baguales en Argentina, La Verdadera Argentina, y sectores más al sur de la misma -Eas. Santa Ana, Cancha Carrera-), también se registran en menor frecuencia hacia el área sureste, que incluye el campo volcánico Pali Aike y los espacios próximos a él. Los resultados indican que varias fuentes potenciales de lutita permiten explicar la distribución de instrumentos laminares en el área noroccidental. En el área suroriental, la presencia de instrumentos laminares podría ser producto de mecanismos de circulación humana y de bienes a escala amplia, o bien del uso de otras materias primas de fuentes de aprovisionamiento cercanas aún no identificadas.

Palabras clave: producción laminar; materias primas líticas; intensidad de uso; distribución espacial; Patagonia sur continental.

\begin{abstract}
A first approach to the distribution of the laminar production manufactured on shale at the south of Baguales ranges (southern continental Patagonia, between Argentina and Chile) is presented. Although blades are characteristic of the northwestern area to the Pali Aike volcanic field population node (Última Esperanza in Chile, and the zone surrouding sierra Baguales in Argentina, La Verdadera Argentina, and sectors further south -Eas. Santa Ana, Cancha Carrera-), they are also recorded less frequently towards the southeast area, which includes the Pali Aike volcanic field and spaces close to it. The results indicate that several potential sources of shale allow explaining the distribution of laminar instruments in the northwestern area. In the southeastern area, the presence of laminar instruments could be a product of large-scale circulation mechanisms of humans and goods, or due to the use of other raw materials from nearby sources that have not been identified yet.
\end{abstract}

Keywords: blade production; lithic raw materials; intensity of use; spatial distribution; southern continental Patagonia.

\section{Introducción}

Junto con otros marcadores arqueológicos, principalmente los motivos rupestres, los valores de isótopos estables sobre restos óseos humanos y los recursos marinos, (Barberena 2008; Borrero y Barberena 2006; Charlin y Borrero 2012), los estudios líticos señalan la existencia de dos diferentes expresiones espaciales de las poblaciones humanas en el sur de Patagonia continental (Argentina y Chile), al menos para el Holoceno tardío (Charlin et al. 2011). En particular, las materias primas principalmente explotadas y las tecnologías implementadas para la manufactura artefactual permiten diferenciar dos grandes áreas al sur de la sierra Baguales (Figura 1):

a) Los sitios del campo volcánico Pali Aike (CVPA) hasta las nacientes del río Gallegos hacia el oeste (Laguna Cóndor) y las porciones adyacentes de la costa atlántica y del estrecho de Magallanes, caracterizados por el predominio de la tecnología de lascas y la explotación de rocas volcánicas de grano fino oscuro tipo Potrok Aike (Charlin 2009, Charlin et al. 2011; Charlin y Pallo 2015). b) Los sitios del área noroccidental al CVPA, incluyendo ambos lados de la frontera internacional (Última 
Esperanza en Chile, y la zona circundante a sierra Baguales en Argentina, La Verdadera Argentina -LVA-, y sectores más al sur de LVA -Eas. Santa Ana, Cancha Carrera-), que se caracterizan por la producción laminar y el uso de rocas sedimentarias, principalmente lutita (Borrazzo 2006, 2008; Langlais y Morello 2009).

De acuerdo con el conocimiento aportado por las investigaciones previas, las láminas son características del área noroccidental al CVPA (área noroccidental en la Figura 1). Sin embargo, también se registran en menor frecuencia en algunos sitios del CVPA y los espacios asociados a él (área suroriental en la Figura 1). Debido a la frecuencia diferencial que presentan las láminas a escala amplia, este marcador arqueológico constituye un indicador de importancia para discutir aspectos vinculados con las conexiones espaciales y la circulación de bienes, que podría involucrar mecanismos de interacción poblacional y/o la superposición de distintos rangos de acción en determinados lapsos. Como un primer acercamiento a esta discusión, el objetivo de este trabajo es evaluar la existencia de una o varias fuentes potenciales de aprovisionamiento de la lutita empleada en la manufactura de los instrumentos laminares registrados al sur de la sierra Baguales, considerando la distribución, tamaño e intensidad de uso de estos artefactos en función de la distancia a las potenciales fuentes.

\section{Producción laminar}

La presencia de producción laminar en el área de estudio se evaluó siguiendo la propuesta tecnomorfológica de Vetrisano (2016). De acuerdo con esta propuesta, los productos laminares serán aquellos artefactos cuyo largo al menos duplique su ancho (definición estricta de laminaridad) y que tengan como mínimo una arista guía, producida por lascados paralelos o convergentes. Esta definición engloba tanto las hojas como las lascas de

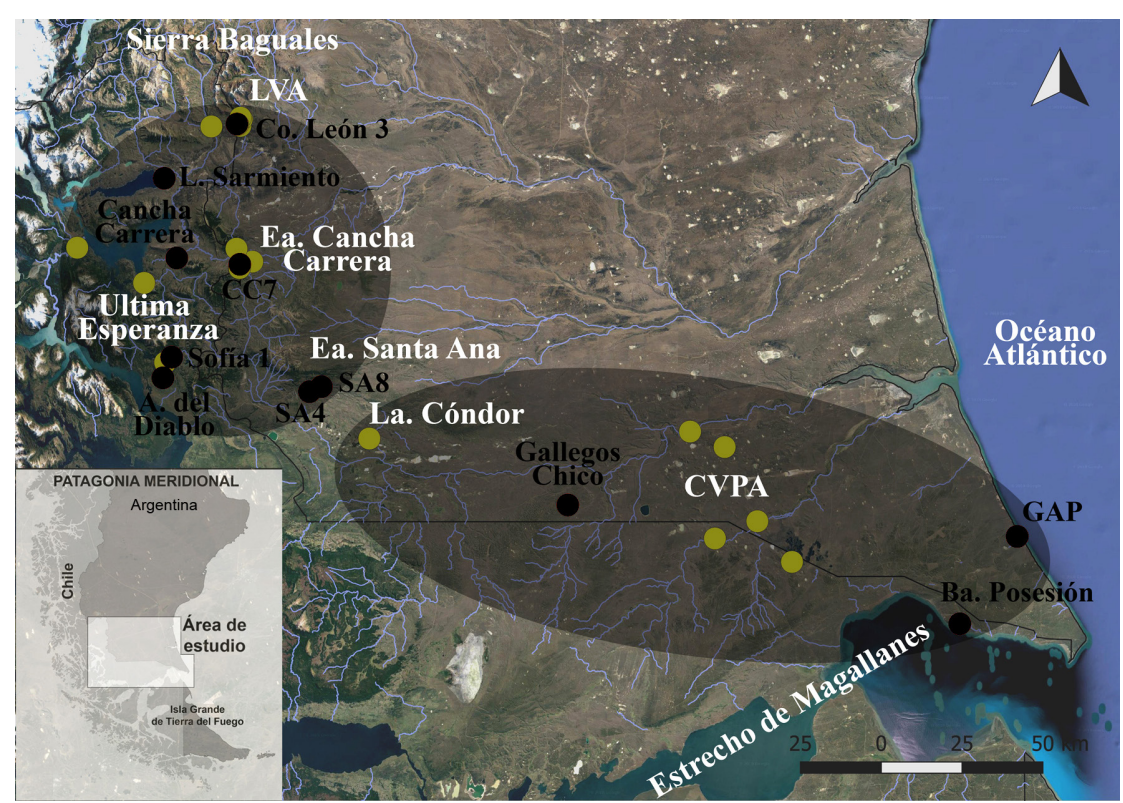

arista de módulo laminar (Aschero 1975, 1983), así como la variedad de terminologías relativas a esta producción en el área de estudio (e.g. Borrazzo 2008; Jackson y Prieto 2005; Langlais y Morello 2009; Ortiz Troncoso 1972; Nami 2009).

La información obtenida procede de relevamientos propios y publicados por otros autores (Borrazzo 2006, 2008; Borrero et al. 1976; Emperaire 1988; Jackson y Prieto 2005; Ortiz Troncoso 1972, ver Figura 1), que incluyeron recolecciones en superficie (selectivas y no selectivas) y en estratigrafía. Las cronologías obtenidas para los artefactos laminares hallados en estratigrafía abarcan diferentes momentos del Holoceno (Borrazzo 2006, 2008; Cardillo et al. 2010; Jackson 2007; Langlais y Morello 2009; Nami 2009; Sanguinetti de Bórmida 1976; Sierpe et al. 2009), incluyendo una aparición temprana en el sitio Sofía 1 de Última Esperanza (10700 a 10100 AP, Jackson y Prieto 2005). De acuerdo con el análisis de artefactos procedentes de conjuntos propios, la baja frecuencia relativa de láminas a escala amplia y la presencia de solo unos pocos núcleos con extracciones laminares aisladas en el área noroccidental (Borrazzo 2008; Emperaire 1988), se sugiere la existencia de una producción laminar asistemática al sur de la sierra Baguales, que sólo implicó la obtención oportunística de estas formas base (Langlais y Morello 2009; Vetrisano 2016).

Debido a la disparidad de la información publicada, la muestra analizada solo incluye datos sobre el largo y ancho de los artefactos formatizados sobre láminas ( $n=29$, incluyendo un $n=20$ para el área noroccidental al CVPA y un $n=9$ para el CVPA y los sectores asociados), permitiendo establecer una comparación de su distribución y grado de reducción a escala amplia. Estos instrumentos suelen ser raederas o cuchillos de grandes dimensiones, en general con filos dobles convergentes, o con un sólo filo largo formatizado, pudiendo incluir filos cortos en su parte proximal o distal (Figura 2). La materia prima lítica sobre la que fueron manufacturados

Figura 1: Distribución de la producción laminar (todos los puntos) y de la muestra de instrumentos laminares considerada en este trabajo (puntos oscuros) para las distintas áreas al sur de sierra Baguales: noroccidental y suroriental (óvalos negros).

Figure 1: Distribution of the blade production (all points) and the sample of blade instruments considered in this paper (dark points) for the different area south of Baguales range: northwestern and south-east (black ovals). 
Figura 2: Variabilidad en las dimensiones de la muestra artefactual. Ejemplos de instrumentos laminares registrados en el área de estudio: 1. Co. León 3 (Borrazzo 2006); 2. Lago Sarmiento (Ortiz Troncoso 1972); 3 y 6. Ea. Santa Ana; 4. Cañadón Gap (Cardillo et al. 2010); 5. Las Buitreras (Sanguinetti de Bórmida 1976).

Figure 2: Variability in dimensions of the artifactual sample. Examples of blade instruments recorded in the study area: 1. Co. León 3 (Borrazzo 2006); 2. Sarmiento Lake (Ortiz Troncoso 1972); 3 and 6. Ea. Santa Ana; 4. Cañadón Gap (Cardillo et al. 2010); 5. Las Buitreras (Sanguinetti de Bórmida 1976).
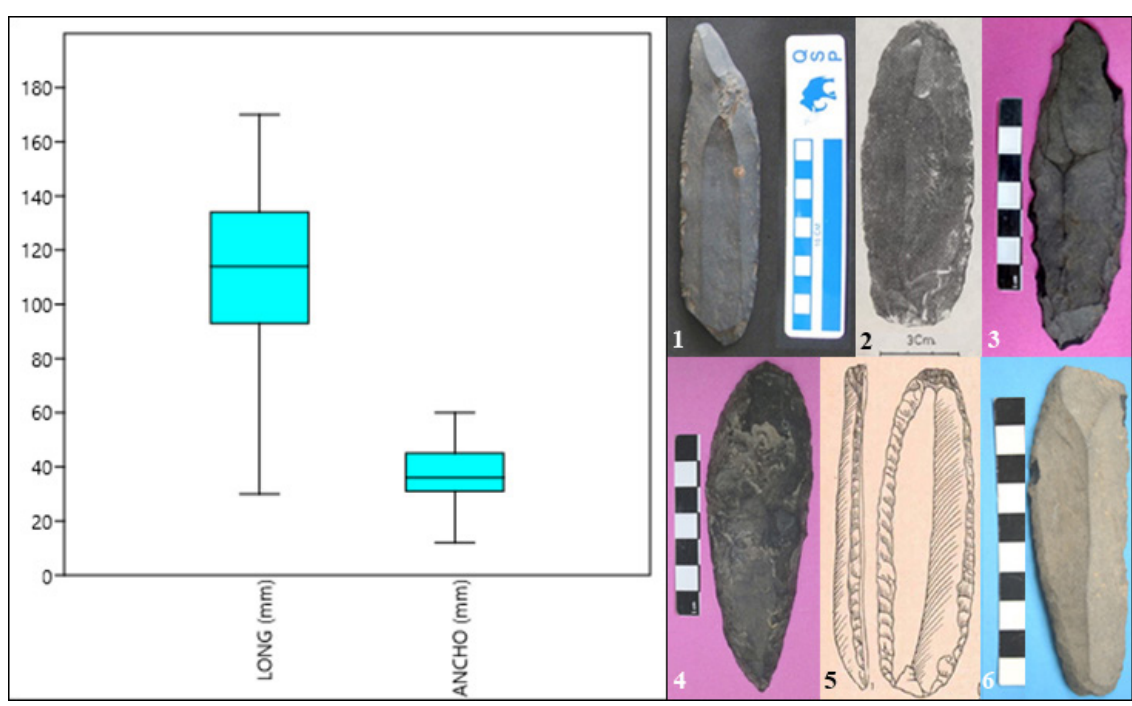

fue clasificada inicialmente como basalto o esquisto (Ortiz Troncoso 1972; Emperaire 1988), aunque las identificaciones posteriores, a través de cortes delgados, las catalogaron como lutitas o rocas lutíticas de color gris muy oscuro, que incluyen lutitas, fangolitas y grauvacas (Borrazzo 2008; Langlais y Morello 2009). En Cañadón Gap, por otra parte, la descripción de las materias primas arrojó interpretaciones dispares (lutita, pelita silicificada o metamórfica).

El tamaño medio de la muestra total alcanza $111 \mathrm{~mm}$ en largo y 37,12 mm en ancho, con largos máximos ocurriendo tanto en LVA, en Cerro León $3(170 \mathrm{~mm})$, como sobre la costa atlántica, en Cañadón Gap (158 mm). El ancho máximo se registra en el área noroccidental, en Lago Sarmiento (60 mm), aunque valores similares también se presentan en Cañadón Gap (50,5 mm) (Figura 2). La distribución espacial de tales dimensiones artefactuales dentro del área de estudio fue considerada a fin de evaluar las potenciales fuentes de procedencia de la materia prima empleada para la manufactura artefactual.

\section{Fuentes potenciales de aprovisionamiento de lutita}

Las principales fuentes potenciales de aprovisionamiento de la lutita se localizan entre LVA y Laguna Larga (Borrazzo 2008; Langlais y Morello 2009; Sierpe et al. 2009; Charlin et al. 2011), donde suele

Figura 3. Fuentes potenciales de lutita (puntos amarillos: bloques de lutita en color oscuro; puntos rosas: nódulos en variados colores, incluyendo negro) y disponibilidad de lutita en LVA (fotografía de K. Borrazzo).

Figure 3. Potential shale sources (light dots: black shale in dark color, black dots: nodules in several colors, including black) and shale availability in LVA (photo by K. Borrazzo) presentarse en forma de gravas y bloques (Figura 3). Esta litología es parte de la formación geológica Cerro Toro, que se extiende de norte a sur en la porción oeste del área de estudio, pudiendo registrarse afloramientos de lutita en las inmediaciones de muchos de los sitios aquí considerados. Sin embargo, hasta el momento, sólo el área de LVA registró la explotación efectiva de lutita a partir de la identificación de núcleos con extracciones laminares aisladas y grandes bloques de calidad apta para la talla (Figura 3) que poseen un tamaño medio de 708, 15 mm (Borrazzo 2008), adecuado para la extracción de láminas grandes. Debido a esto y ante la falta de datos similares para las otras fuentes potenciales del sector oeste, LVA podría representar el principal sector de obtención de la lutita empleada para manufacturar los artefactos laminares registrados al sur de la sierra Baguales. Cabe señalar que la lutita disponible en el CVPA se presenta en forma de nódulos pequeños, de variados colores, con un tamaño medio de $78,87 \mathrm{~mm}$, y calidades inferiores para la talla (Charlin 2009; Charlin y Pallo 2015), desalentando la posibilidad de su utilidad para la obtención de las formas base laminares observadas a escala amplia, cuyos largos sobrepasan estas dimensiones en la mayoría de los casos.

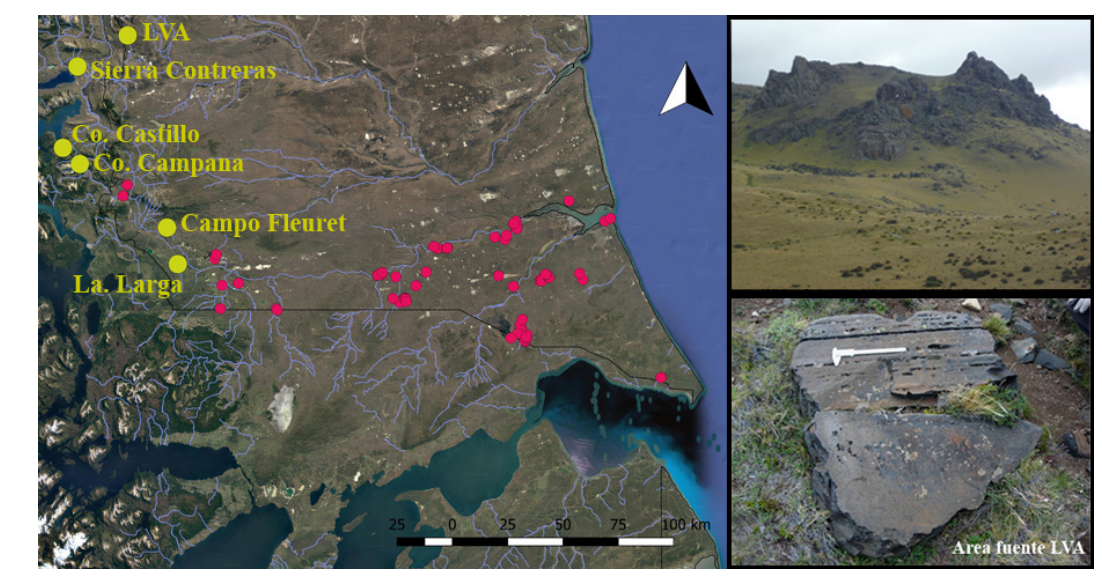




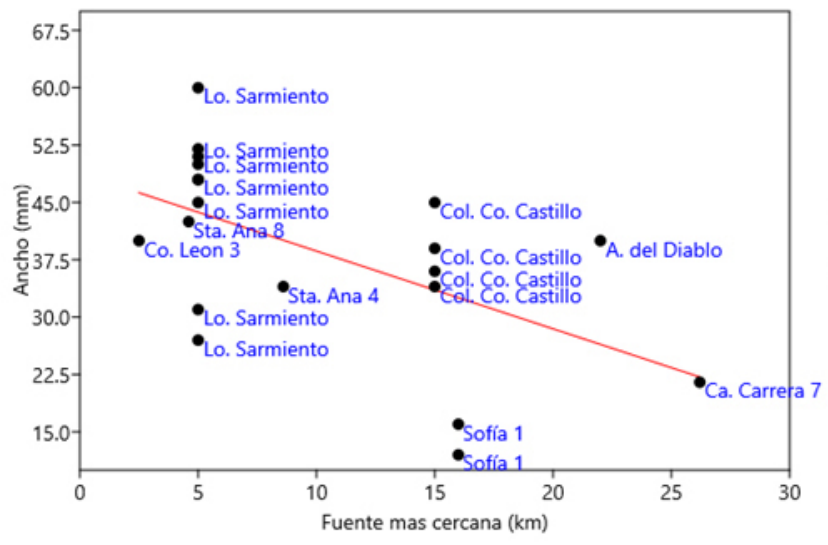

Figura 4: Análisis de regresión para contrastar la H2.

Figure 4: Regression analysis to test $\mathrm{H} 2$.

De acuerdo con esta información se plantearon dos hipótesis de trabajo: 1. El sector LVA es la única fuente de aprovisionamiento de la lutita empleada como forma base de los instrumentos laminares del área de estudio; y 2. Existieron varias fuentes de aprovisionamiento de la lutita empleada como forma base de los instrumentos laminares del área de estudio. Ambas hipótesis fueron contrastadas evaluando el ajuste de un modelo de regresión lineal, considerando que el tamaño de los instrumentos disminuirá a medida que aumenta la distancia a la fuente de aprovisionamiento. En estos casos, la distancia puede representar un proxy de los costos del aprovisionamiento y transporte de rocas, quedando reflejado en la intensidad de uso o reducción del artefacto, dado que la misma afecta las dimensiones artefactuales (Dibble 1984). Aquí, el largo y ancho de los instrumentos laminares son considerados como variables dependientes respecto de la distancia al área fuente LVA (Cerro Tridente') $\left(H_{1}\right)$ y luego respecto de las fuentes potenciales de lutita registradas hasta el momento $\left(\mathrm{H}_{2}\right)$, con el fin de evaluar el mejor ajuste del modelo de regresión.

\section{Resultados}

El análisis de regresión para la $\mathrm{H}_{1}$ no arrojó valores significativos $(F=0.1027$ y permutat. $p=.75$ para el largo; $\mathrm{F}=1.677$ y permutat. $p=.21$ para el ancho, con 9999 permutaciones) en la variación del tamaño de los instrumentos $(n=29)$ respecto del Cerro Tridente, lo cual indica que no hay una relación de dependencia entre ambas variables (distancia a la fuente/tamaño de las láminas). Una segunda regresión para esta hipótesis, se realizó sin los 9 artefactos pertenecientes a los sitios del área suroriental (Gallegos Chico, Bahía Posesión y Cañadón Gap), a fin de evaluar si la existencia de diferentes rangos de acción influía en el análisis. Sin embargo, el largo ( $\mathrm{F}=2.655$; permutat. $p=.12,9999$

1 Cabe aclarar que por cuestiones operativas se toma aquí al Cerro Tridente para medir las distancias al área fuente LVA.

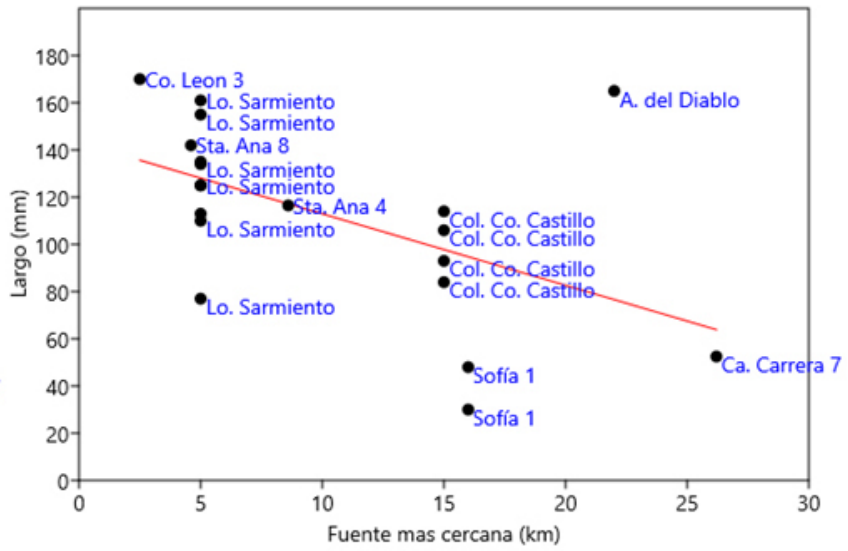

permutaciones) y ancho ( $\mathrm{F}=3.461$; permutat. $p=.08$, 9999 permutaciones) de los instrumentos tampoco varía significativamente en función de la distancia al Cerro Tridente. Por lo tanto, este último no habría funcionado como la única fuente de aprovisionamiento de la lutita empleada en la producción laminar del área de estudio.

Con el mismo procedimiento señalado anteriormente se testeó la $\mathrm{H}_{2}$. En este caso, la segunda regresión $(n=20)$, que excluye los instrumentos del área suroriental, arrojó valores estadísticamente significativos, tanto para la variación en el largo ( $F=6.91$; permutat. $p=.02,9999$ permutaciones) como en el ancho $(F=8.16$; permutat. $p=.01,9999$ permutaciones) de los instrumentos laminares (Figura 4), aunque los porcentajes de varianza explicada son moderados en ambos casos $\left(R^{2}=.28, R^{2}=\right.$ .31 respectivamente). Mientras que el largo disminuye $3 \mathrm{~mm}$ por $\mathrm{km}$ a la fuente más próxima al lugar de descarte, el ancho decae $1 \mathrm{~mm}$ por $\mathrm{km}$. Esto indicaría que existieron varias fuentes de aprovisionamiento de lutita seleccionadas para la manufactura, probablemente vinculadas de manera relativamente próxima con cada uno de los espacios de hallazgo de los instrumentos laminares dentro del área noroccidental.

En consideración a los instrumentos laminares recuperados en el área suroriental, recientemente se presentaron diferencias en la identificación petrográfica de las muestras de Cañadón Gap. Según los análisis, las mismas podrían ser pelitas silicificadas o pizarras (rocas metamórficas de grado bajo), teniendo estas últimas su más cercano lugar de procedencia en la costa del Estrecho de Magallanes, a $35 \mathrm{~km}$ de distancia lineal de Cañadón Gap. Sin embargo, los nódulos de pizarra registrados hasta el momento en el Estrecho (Charlin y D'Orazio 2015) son más pequeños que los instrumentos del sitio. Por lo tanto, la propuesta de una presencia de instrumentos laminares más grandes de lo esperado en el área suroriental producto de mecanismos de circulación 
humana y de bienes a escala amplia es, por el momento, la opción más probable.

\section{Discusión y conclusiones}

Los resultados indican que las fuentes potenciales de lutita conocidas hasta el momento en el área noroccidental al CVPA permiten explicar la distribución de los artefactos laminares al sur de la sierra Baguales de forma más ajustada que considerando una sola fuente de aprovisionamiento (LVA - Cerro Tridente). Aunque, hasta el momento, sólo en el área de LVA se registró la explotación efectiva de lutita y en el resto de los sectores son identificadas fuentes sin explotación. Debido a esto último, no se descarta que la falta de significancia estadística pueda ser insuficiente para refutar la hipótesis relativa a LVA - Cerro Tridente como única fuente (Amrhein et al. 2019) o que existan otras fuentes potenciales dentro del área noroccidental al CVPA. Pero la amplitud de la formación Cerro Toro y los escasos productos laminares en el área de estudio, dificultan su reconocimiento, así como la recuperación de productos de actividades extractivas en tales fuentes, que indiquen su uso efectivo (e.g. núcleos con extracciones laminares).

Por su parte, los instrumentos laminares del área suroriental son llamativamente grandes en comparación con los nódulos de lutita disponibles en las fuentes potenciales del CVPA y los espacios asociados a él (Charlin 2009; Charlin y Pallo 2015). Esto mantiene la propuesta de los estudios comparativos sobre la tecnología lítica y otrosmarcadores arqueológicos (Barberena 2008; Borrero y Barberena 2006; Charlin et al. 2011; Charlin y Borrero 2012), acerca de la existencia de distintos nodos poblacionales con diferente expresión espacial en el sur de Patagonia continental (Argentina y Chile). La presencia de instrumentos laminares en sitios del área suroriental (Gallegos Chico, Cañadón Gap y Bahía Posesión), por lo tanto, podría ser un producto de mecanismos de circulación humana y de bienes a escala amplia, que pudo involucrar la superposición de los distintos rangos de acción previamente identificados al sur de la sierra Baguales. Sin embargo, resta evaluar si existió un uso de otras materias primas líticas, como las pizarras procedentes de la costa norte del estrecho de Magallanes, que podrían ser confundidas con lutitas a ojo desnudo. La combinación de futuros estudios petrográficos y análisis de rayos $X$ permitirá evaluar de forma más robusta si la/s fuente/s potencial/es de procedencia de la roca empleada en la manufactura de los instrumentos laminares del área suroriental corresponden a rocas sedimentarias físiles (e.g. lutitas) o metamórficas de bajo grado (e.g. pizarras), a partir de la determinación de las asociaciones minerales, las microestructuras y el índice de cristalinidad de la illita (Oriolo com. pers. 2018). La agenda futura también implicará revisar la colección de artefactos y tomar otras medidas de intensidad de uso, a fin de establecer comparaciones más precisas sobre la producción laminar en el extremo sur de Patagonia Meridional.

\section{Agradecimientos}

Puerto Madryn, 6 de junio de 2019.

Los comentarios y sugerencias de los evaluadores contribuyeron a mejorar la versión final de este trabajo. Esta investigación ha sido financiada por la ANPCyT (PICT 2014-2061) y el CONICET.

\section{Referencias citadas}

Amrhein, V., Greenland, S. y McShane B. (2019). Retire statistical significance. Nature 567, 305-307.

Aschero, C. (1975/1983). Ensayo para una clasificación morfológica de los artefactos líticos aplicada a estudios tipológicos-comparativos. Informes en CONICET.

Barberena, R. (2008). Arqueología y biogeografía humana en Patagonia Meridional. SAA, Buenos Aires.

Borrazzo, K. (2006). Tecnología lítica del alero Cerro León 3 (Santa Cruz, Argentina). Magallania 32, 63-74.

Borrazzo, K. (2008). Análisis tecnológico de distribuciones artefactuales en la periferia sudeste de la Sierra Baguales (Santa Cruz). Magallania 36 (1), 103-116.

Borrero, L.A. y Barberena, R. (2006). Hunter-Gatherer home ranges and marine resources. An archaeological case from Southern Patagonia. Current Anthropology 47(5), 855-867.

Borrero, L.A, Crivelli, E. y Mengoni, G. (1976). Investigaciones arqueológicas en el sitio "Alero del diablo". Anales del Instituto de la Patagonia 7, 75-85.

Cardillo, M., Charlin, J. y Borrazzo, K. (2010). Una exploración de la variación métrica y morfológica en instrumentos de filo largo en Patagonia meridional. La arqueometría en Argentina y Latinoamérica, pp. 147152. Córdoba: Editorial de la FFyH, UNC.

Charlin, J. (2009). Estrategias de aprovisionamiento y utilización de las materias primas líticas en el campo volcánico Pali Aike. Oxford, Inglaterra: Archaeopress.

Charlin, J. y Borrero, L.A. (2012). Rock Art, Inherited Landscapes and Human Populations in Southern Patagonia. En McDonald y P. Veth (Eds.), A Companion to Rock Art, part IV, J. pp. 381-398. New Jersey: WileyBlackwell.

Charlin, J., Borrero, L.A. y Pallo, M.C. (2011). Ocupaciones humanas en el área noroccidental del río Gallegos (Prov. Santa Cruz, Argentina). En L. Borrero y K. Borrazzo (Eds.), Bosques, montañas y cazadores. Investigaciones arqueológicas en Patagonia meridional, pp. 179-210. 


\section{Buenos Aires: CONICET-IMHICIHU.}

Charlin, J., y D'Orazio, M. (2015). Disponibilidad de materias primas líticas en la costa nororiental del estrecho de Magallanes (Chile). Una aproximación exploratoria. Magallania 43, 133-154. doi: 10.4067/ S0718-22442015000200007

Charlin, J., y Pallo, M.C. (2015). Disponibilidad de rocas y costos de aprovisionamiento en el extremo austral de Patagonia meridional: integración de resultados en una escala regional. Intersecciones en Antropología Volumen Especial 2, 125-138.

Dibble, H.L. (1984). Interpreting typological variation of Middle Paleolithic scrapers: function, style, or sequence of reduction? Journal of Field Archaeology 11, 431-436.

Emperaire, J. (1988). Paisajes y humanos prehistóricos de la Patagonia. Anales del Instituto de la Patagonia $18,79-94$

Jackson, D. (2007). Estructura, intensidad y reiteración en las ocupaciones paleoindias en cuevas y aleros de Patagonia Meridional (Chile). Cazadores Recolectores del Cono Sur 2, 67-87.

Jackson, D y Prieto, A. (2005). Estrategias tecnológicas y conjunto lítico del contexto paleoindio de Cueva Lago
Sofía 1, Última Esperanza. Magallania 33(1), 115-120.

Langlais, M. y F. Morello (2009). Estudio tecno-económico de la industria lítica de Cerro Castillo (Última Esperanza, Chile). Magallania 37 (1), 61-84.

Nami, H.G. (2009). Avances de las investigaciones arqueológicas en la localidad arqueológica de Pali Aike, extremo sur de la provincia de Santa Cruz. En Estado actual de las investigaciones realizadas sobre Patrimonio Cultural en Santa Cruz, pp. 235-241. Río Gallegos: Subsecretaría de Cultura, Gobierno de Santa Cruz.

Ortiz Troncoso, O. (1972). Material lítico de Patagonia austral. Seis yacimientos de superficie. Anales del Instituto de la Patagonia 3, 49-102.

Sanguinetti de Bórmida, A. (1976). Excavaciones prehistóricas en la cueva de "Las Buitreras" (provincia de Santa Cruz). Relaciones X, 271-292.

Sierpe, V., Prieto, A., Huidobro, C. y Stern, C. (2009). Excavaciones Arqueológicas en el sitio "Alero Quemado" (Última Esperanza, Magallanes, Chile). Magallania 37(2), 177-189.

Vetrisano, L. (2016). Producción laminar en la cuenca del río Santa Cruz. Actas del XIX CNAA, pp. 807-809, 8 al 12 de agosto, Tucumán. 